\title{
Oscillator seeding of a high gain harmonic generation free electron laser in a radiator-first configuration
}

\author{
P. Gandhi \\ University of California, Berkeley, California 94720, USA \\ G. Penn* and M. Reinsch \\ Lawrence Berkeley National Laboratory, Berkeley, California 94720, USA \\ J.S. Wurtele \\ University of California, Berkeley, California 94720, USA, \\ and Lawrence Berkeley National Laboratory, Berkeley, California 94720, USA \\ W. M. Fawley \\ Sincrotrone Trieste, Italy \\ (Received 20 November 2012; published 6 February 2013)
}

\begin{abstract}
A longitudinally and transversely coherent, high repetition rate $\mathrm{x}$-ray source with widely tunable wavelength is desired for a variety of experimental applications. A free electron laser (FEL) powered by an electron beam from a superconducting linac can reach the desired peak and average $\mathrm{x}$-ray power levels with transverse coherence. However, generating longitudinally coherent $\mathrm{x}$-ray pulses is a significant challenge, especially at high repetition rate. This paper presents a one-dimensional theoretical and numerical investigation of a method to achieve longitudinal coherence and high repetition rate simultaneously. We propose a "radiator-first" configuration, wherein an FEL oscillator follows a high gain harmonic generation (HGHG) FEL. The oscillator generates seed power that is directed upstream to initiate the HGHG process in a following electron bunch. This configuration allows for the generation of radiation at short wavelength, which is highly sensitive to energy spread, to occur before the longer wavelength oscillator, whose performance is not seriously degraded by the beam heating in the upstream radiator. The dynamics and stability of this radiator-first scheme is explored analytically and numerically. A single-pass, 1D map is derived using a semianalytic model for FEL gain and saturation. Iteration of the map is shown to be in good agreement with simulations. A numerical example is presented for a soft x-ray FEL.
\end{abstract}

DOI: 10.1103/PhysRevSTAB.16.020703

PACS numbers: $41.60 . \mathrm{Cr}$

\section{INTRODUCTION}

Superconducting linear accelerators (sc linacs) operating in continuous wave (cw) mode have the ability to produce high quality electron beams with bunch repetition rates of $1 \mathrm{MHz}$ and greater [1]. A free electron laser (FEL) facility based on such a linac could power multiple FELs, reach greater pulse-to-pulse stability, and has the potential to realize much greater scientific throughput than would a room temperature system operating at $\sim 1 \mathrm{kHz}$ or less. Furthermore, many experiments require a high average flux together with a nondestructive peak flux; a cw sc FEL is ideally suited to provide such pulses. X-ray pulses with longitudinal coherence and/or high spectral density are also needed by many experiments. This last requirement has

\footnotetext{
*gepenn@lbl.gov

Published by the American Physical Society under the terms of the Creative Commons Attribution 3.0 License. Further distribution of this work must maintain attribution to the author(s) and the published article's title, journal citation, and DOI.
}

motivated consideration of a wide variety of seeding schemes [2], such as self-seeding [3], high gain harmonic generation (HGHG) [4], echo-enabled harmonic generation (EEHG) [5], and direct seeding. The latter three schemes require external, coherent, high repetition rate laser sources. The performance of HGHG schemes at very high harmonics is very sensitive to electron beam properties such as incoherent energy spread, temporal quadratic chirps in the mean energy, and transverse emittance. Laser-driven sources using high harmonic generation (HHG) can produce the short wavelengths required to seed soft $\mathrm{x}$ rays through lower $(\sim 10$ th) harmonic HGHG FELs, but they are not now, nor are they likely to be in the foreseeable future, viable at $\mathrm{MHz}$ or higher repetition rates. FEL output at high harmonics of an external UV seed may be possible through EEHG, but such has not yet been demonstrated experimentally; moreover, the seed lasers would still need to operate at high repetition rate. The development of a reliable seed for EEHG is easier at longer wavelengths but this requires operation at higher harmonics. Self-seeding is a promising scheme in that it does not require anything beyond optics, a chicane, and the electron bunch, but it requires a significant 
increase in undulator length beyond what is required to reach saturation for a pure SASE configuration. The only experimental test so far of self-seeding [6] exhibited some limitations in terms of shot-to-shot variations, possibly due to the specific operating conditions at LCLS.

This paper proposes a different configuration that uses the electron bunches to power an FEL oscillator whose output pulses seed an HGHG FEL. These pulses would be highly coherent, have short wavelengths comparable to HHG sources, and would be capable of matching any repetition rate delivered by the accelerator. The FEL oscillator would operate at wavelengths sufficiently long where reasonably reflective mirrors can be fabricated but at wavelengths short enough (e.g., in the extreme UV regime) that the harmonic upshift needed to reach the soft $x$-ray regime is not excessive. The novelty in the scheme being considered is that the oscillator follows the radiator, so that the high quality input electron beam is first used for the most difficult task from the standpoint of emittance and energy spread requirements: namely, radiation generation at the shorter wavelength. The spent electron beam from the radiator is then used to power the oscillator. As for all oscillators, there is some start-up period where the first bunches in a pulse train build up fields in the cavity to an equilibrium value, for which the HGHG seeding scheme will be tuned.

The idea of placing the oscillator at the end of the beam line has been considered before [7]; however, this work did not explore the possibility of harmonic generation and the geometry was not a critical feature for achieving good performance. Electron bunches were divided into two groups, those which radiate well in the radiator section but not the oscillator, and conversely those which perform poorly in the amplifier due to a weak input seed but well in the oscillator. Placing the radiator in front of the oscillator was used primarily as a clever way to avoid having to kick alternating electron bunches along different paths. In the HGHG context, later research [8] focused on using an oscillator directly as the modulation stage of an HGHG FEL. However, the oscillator created significant energy spread which hampered the downstream amplifier, and this idea does not seem to have been further pursued.

Wurtele et al. [9] reintroduced the radiator-first geometry as one of several proposed FEL configurations for echoenabled harmonic generation (EEHG). The major advantage of EEHG over HGHG is its ability to reach much higher harmonics of the seed radiation, and therefore shorter wavelengths, in a single stage. Furthermore, since the target harmonic is adjustable but needs to be an integer, going to higher harmonics allows for finer tunability in the output radiation and requires a smaller tuning range in the oscillator for continuous tuning. The EEHG concept, however, requires two input radiation pulses with a high level of stability [10] instead of just the one seed required by HGHG. The EEHG scheme also has several beam physics constraints and technological complexities greater than those of the HGHG system. In view of all these considerations, here we consider the simpler-and analytically more tractable-HGHG radiator-first configuration. Because the useful tuning range of multilayer mirrors for short wavelengths is typically of the order of a few percent [11], this simplification may incur the cost of losing continuous tuning between harmonics through tuning of the oscillator.

In this paper, we first describe the HGHG radiator-first scheme and then develop a one-dimensional nonlinear map to model the evolution of this system. The derivation uses well-known FEL formulas regarding HGHG and oscillator FEL operation, along with simplifying assumptions, to express the output power of bunch $k$ as a function of the output power of bunch $(k-1)$ and various system parameters (e.g., the FEL parameter, mirror losses, energy spread, etc.). The results of this map are compared to time-independent, onedimensional simulations for a soft x-ray example. A stable operating point is found, illustrating the potential for the radiator-first configuration to yield a highly uniform train of $\mathrm{x}$-ray pulses. The analysis confirms one-dimensional simulation results and yields useful expressions for quickly finding workable parameters; it also provides insight into the system's dynamics and stability. Detailed two-dimensional time-dependent simulations would be useful for systematic studies, but in this paper we focus on qualitative operational features, such as the ability to attain a stable operating point and typical peak power levels.

\section{THE RADIATOR-FIRST SCHEME}

The major motivation for considering the type of scheme diagramed in Fig. 1 is that it eliminates the need for an external, high repetition rate seed laser. Since the electrons

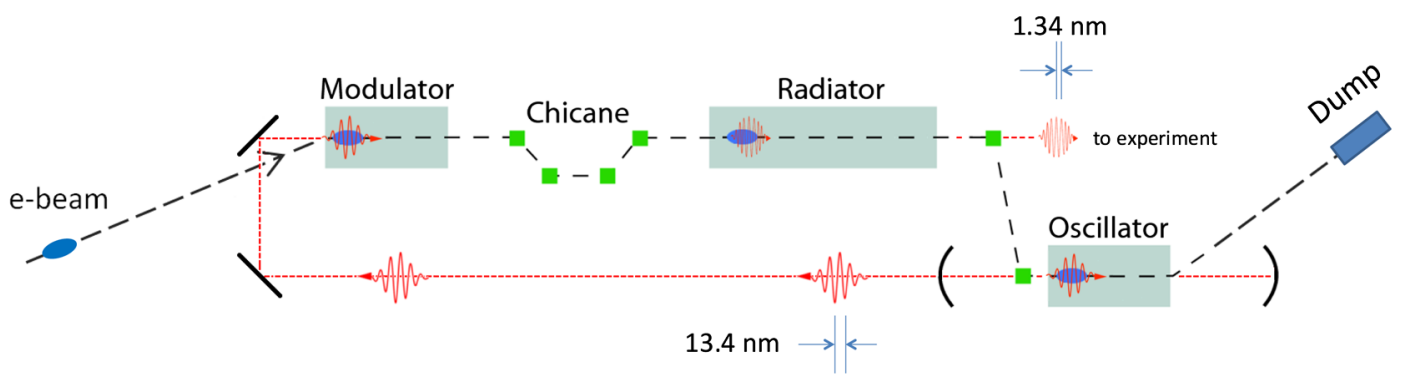

FIG. 1. A diagram of the HGHG "radiator-first" scheme. 
are doing all the work of generating the radiation for both the seeding and the user beam lines, the spacing between electron bunches is now the limiting factor for the FEL repetition rate. This comes at the cost of added complications associated with the oscillator and the transport of radiation from the oscillator back upstream to the modulator. The main use for such a beam line is for wavelengths too short for multilayer mirrors but too long for effective Bragg scattering. Another motivation to run the oscillator at a subharmonic would be to reduce the length of undulator required for sufficient gain. Others have considered similar schemes in which the oscillator is used to replace the modulator, and is located before the radiator $[8,12,13]$. This may seem like a more attractive option, since there is no need to transport radiation or worry about synchronizing the radiation pulse with the electron bunch at two different locations. It is, however, very difficult to control the saturation level of an oscillator in order that the beam does not come out overbunched and with too large an energy spread. While a transverse optical klystron configuration $[9,13]$ appears to be a promising means to control the saturation power, there is no need for this in the radiator-first scheme. We make no use of the electron bunch after exiting the oscillator, avoiding the need to fight its natural tendency to extract as much energy from the beam as possible.

An electron bunch entering the HGHG radiator-first FEL begins by passing through the modulator section and a chicane, followed by the radiator section and, finally, by the oscillator. In the modulator, the electrons develop an energy modulation under the effect of radiation which has been outcoupled and transported from the oscillator at the end of the system. This radiation was produced after the passage of the previous bunch, or from an earlier bunch, depending upon the particular timing requirements. A chicane transforms this energy modulation into a coherent, periodic density modulation, just as in the conventional implementation of HGHG. This density modulation contains Fourier components at harmonics of the seed radiation; the selected harmonic (in our example we will consider the harmonic $n=10$ ) is used to initiate the FEL process in the radiator section. The resulting radiation pulse is delivered to the user and, given the imposed coherent bunching, this radiation will be nearly fully longitudinally coherent.

The electron bunch, which would normally be discarded after the radiator, is instead then sent downstream to the oscillator. Because the radiator section is tuned to a harmonic of the oscillator that follows, the efficiency and energy bandwidth of the radiator section will be lower than that in the much longer wavelength oscillator, as characterized by their respective FEL parameters. Thus, even if the radiator section reaches FEL power saturation, the quality of the electron beam which enters the oscillator will in general still be sufficient for the oscillator to function effectively. At the start of the radiator section, the electron beam will have a large bunching at the oscillator wavelength, as part of the HGHG scheme to produce bunching at the desired harmonic. It is possible for some of this bunching to persist all the way through to the oscillator, where it could improve the performance. Here we will assume, however, that due to energy dispersion all bunching is completely lost as the beam is transported from the radiator to the oscillator. Our simulations actually include a strong chicane after the radiator to produce this dispersion. The strength of this chicane will have to scale with the oscillator wavelength.

The electron bunch then enters the oscillator. At the end of the oscillator, the electron beam is discarded and the outcoupled radiation is transported back to the modulator to interact with the next electron bunch. The cycle is repeated, and hopefully settles on a stable operating point where the output from pass to pass reaches a steady state. The map described in the next section provides a method to evaluate the dynamics of the entire system and to determine if a stable steady state is achievable for a given set of parameters.

Depending on the parameters and actual layout of the device, it may not be possible temporally to overlap the radiation outcoupled from the oscillator following one bunch to the electron bunch which immediately follows. This problem can be overcome by having multiple radiation pulses within the oscillator cavity. The analysis can be readily extended to this case.

\section{DERIVATION OF THE NONLINEAR MAP}

In order to understand the dynamics and stability of the radiator-first scheme, we must be able to properly model the dependence of the oscillator gain on the energy spread of the beam as it enters the oscillator, to characterize the impact of the resulting power fluctuations on the harmonic generation scheme, and to understand the feedback between the two systems. We make use of a semianalytical model of FEL gain and saturation based on the work of Dattoli et al., [14], to predict the output of the oscillator in a given pass based on the initial energy spread. By coupling this model to an idealized analysis of HGHG [15], we can produce a map that predicts the intensity at the end of the oscillator in a new pass based on the intensity of the previous pass. While this model is one dimensional and does not include pulse propagation effects, it could be extended to include more physics using the work of Dattoli and others. We are also evaluating the use of the more complete analytical theory for short-pulse FEL oscillators developed by Piovella et al. [16] to the study of these types of schemes. We note that the limitation to short-pulse oscillators is not highly restrictive since high repetition rates and high peak currents naturally lead to low charge per bunch and short bunches. On the other hand, once the bunch length of the electron beam is shortened to several FEL cooperation lengths of the radiator, we enter the single spike SASE regime where longitudinal coherence will be guaranteed and all the gymnastics of this 
scheme are unnecessary. Thus, the short-pulse theory is best suited for the intermediate region where the bunch length is less than the slippage length in the oscillator, but longer than the coherence length in the radiator.

\section{A. Logistic model for FEL amplification including saturation}

The model for the FEL process in the undulators of the oscillator section used in this paper is based on a modified version of the standard logistic map which is useful for power amplifiers, and which can be derived as a solution to a self-consistent expansion of the FEL equations in certain limits [17]. Here, we use a semianalytic fit for the numerical parameters, matching the initial conditions and the expected saturation levels, resulting in a model logistic function for high gain:

$$
I(z)=I_{0} \frac{\exp (\Gamma z)}{1+\frac{I_{0}}{I_{\mathrm{sat}}}[\exp (\Gamma z)-1]} .
$$

Here $I_{0}$ is the initial intensity, $I_{\text {sat }}$ is the nominal intensity at saturation, and $\Gamma$ is the growth rate. In the one-dimensional limit the FEL power growth rate is $\Gamma=2 \sqrt{3} k_{w} \rho$, where $k_{w}=2 \pi / \lambda_{w}$ is the undulator wave number, $\lambda_{w}$ is the undulator period, and $\rho$ is the one-dimensional FEL parameter defined as

$$
\rho=\left[\frac{1}{4 \pi} \frac{I_{e}}{I_{A}}\left(\frac{a_{w}[J J]}{1+a_{w}^{2}}\right)^{2} \frac{\gamma_{0} \lambda^{2}}{\Sigma_{A}}\right]^{1 / 3}
$$

The electron beam has peak current $I_{e}$, nominal energy $\gamma_{0}$, energy spread $\sigma_{0}$ at the start of the modulator, and crosssectional area $\Sigma_{A}$ (defined to be $2 \pi \sigma_{x} \sigma_{y}$, so that the current density on axis is $I_{e} / \Sigma_{A}$ for a Gaussian transverse profile). The dimensionless $\mathrm{rms}$ undulator parameter is $a_{w}=e B_{w} / \sqrt{2} k_{w} m_{e} c,[J J]=J_{0}(\xi)-J_{1}(\xi)$ is the coupling parameter for planar undulators with $\xi=a_{w}^{2} / 2\left(1+a_{w}^{2}\right)$, the resonant radiation wavelength is $\lambda=\lambda_{w}\left(1+a_{w}^{2}\right) / 2 \gamma_{0}^{2}$, and $I_{A} \equiv 4 \pi \epsilon_{0} m_{e} c^{3} / e \simeq 17045 \mathrm{~A}$ is the Alfvén current.

This logistic function has been used [18] to generate a map of the oscillator intensity as a function of pass number; we decompose this into separate FEL gain and radiation transport maps in order to incorporate the impact of the HGHG stage as well. The logistic function matches reasonably well with one-dimensional simulations of an oscillator as shown in Fig. 2, and provides a simple means for including both the effects of previous passes and of the electron beam properties.

The one-dimensional saturation intensity of the FEL can be expressed in terms of $\rho$ as $I_{\text {sat }}=c_{f} \rho I_{\text {beam }}$, where $I_{\text {beam }}=\gamma_{0} m_{e} c^{2} I_{e} / e \Sigma_{A}$ is the peak power intensity of the electron beam in the oscillator and $c_{f}=1.6$ yields a good numerical fit [19] to simulation results when 3D effects are weak and the undulator parameter is optimized.

\section{B. Corrections to the linear gain rate from 3D effects}

While our model is essentially one dimensional, the onedimensional FEL parameter $\rho$ is not quite the correct parameter to use in our oscillator configuration because this ignores the effects of incoherent energy spread and the partial overlap between the radiation mode and the electron beam. The dependence of the growth rate on energy spread is a crucial aspect of this analysis, because the energy spread in the oscillator is affected by the FEL performance of the upstream amplifier. The effect of diffraction will be treated as a static, fixed term, but it has a significant quantitative impact on the growth rate because the length scale for diffraction, which is roughly the Rayleigh range for the beam spot size or $2 \Sigma_{A} / \lambda$, can be comparable to or even shorter than the FEL gain length.

To account for these effects, we consider the semianalytic fit for the growth rate obtained by Xie in Ref. [20], where $\Gamma_{3 \mathrm{D}}=\Gamma_{1 \mathrm{D}} /\left(1+\eta_{T}\right)$ and the dimensionless quantity $\eta_{T}$ is a function of scaled electron properties for diffraction, energy spread, and emittance. Here, the effect of emittance is neglected. The dimensionless parameter for diffraction used in Ref. [20] is

$$
\eta_{d}=\frac{\lambda}{2 \Gamma_{1 \mathrm{D}} \Sigma_{A}}=\frac{\lambda \lambda_{w}}{8 \pi \sqrt{3} \rho \Sigma_{A}} .
$$

For the sample parameters discussed below, $\eta_{d}=1.09$ and diffraction alone contributes 0.47 to $\eta_{T}$ in the oscillator. It is difficult to get the diffraction parameter for the oscillator significantly below unity in this scheme.

Instead of directly using the quantity $\eta_{T}$, we attempt to separate out the effects of diffraction and energy spread by defining an FEL parameter $\rho_{2}=\rho /\left(1+\eta_{T}\right)$ which only includes the effect of diffraction, and thus for our purposes will remain fixed from pass to pass. A simple model for the effect of diffraction is to assume that the mode size of the radiation, denoted $\Sigma_{\text {eff }}$, replaces the size $\Sigma_{A}$ of the electron beam in Eq. (2). This yields $\rho_{2}=\rho\left(\Sigma_{A} / \Sigma_{\text {eff }}\right)^{1 / 3}$. The fit from Xie when only diffraction is important is $\eta_{T} \simeq$ $a_{1} \eta_{d}^{0.57}$, where $a_{1}=0.45$. Noting that $\rho$ scales like $\Sigma_{A}^{-1 / 3}$ and $\eta_{d}$ scales like $\Sigma_{A}^{-2 / 3}$, the quantity $\rho / \eta_{T}$ is almost independent of $\Sigma_{A}$ and we can make it completely independent by approximating $\eta_{T}=a_{1} \eta_{d}^{1 / 2}$, yielding

$$
\rho_{d} \equiv \frac{\rho}{a_{1} \eta_{d}^{1 / 2}}=\left[\frac{2 \sqrt{3}}{a_{1}^{2}} \frac{\gamma_{0} \lambda}{\lambda_{w}} \frac{I_{e}}{I_{A}}\left(\frac{a_{w}[J J]}{1+a_{w}^{2}}\right)^{2}\right]^{1 / 2},
$$

and

$$
\Sigma_{\mathrm{eff}}^{1 / 3}=\Sigma_{A}^{1 / 3} \rho / \rho_{2}=\Sigma_{A}^{1 / 3}\left(1+a_{1} \eta_{d}^{1 / 2}\right) .
$$

Note that $\rho_{d}$ is independent of the electron beam size, and, unlike the one-dimensional FEL parameter, scales as beam current to the $1 / 2$ power. This scaling is expected for beams where diffraction has an overwhelming effect. This is only an approximation to more complicated behavior for diffraction-dominated beams [21], but the resulting gain length and mode size are fairly accurate so long as $\eta_{d}$ is 


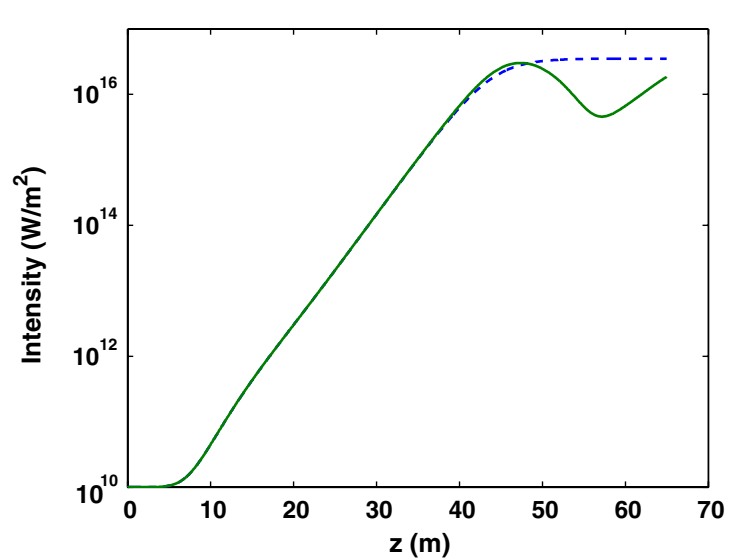

(a)

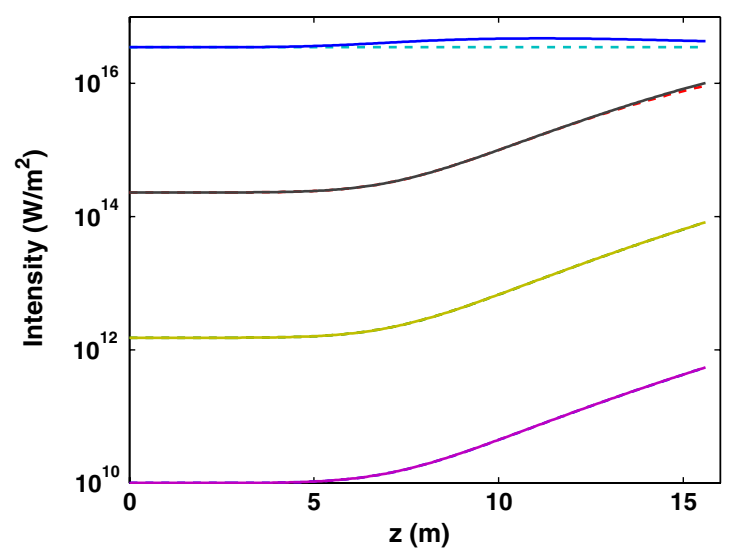

(c)

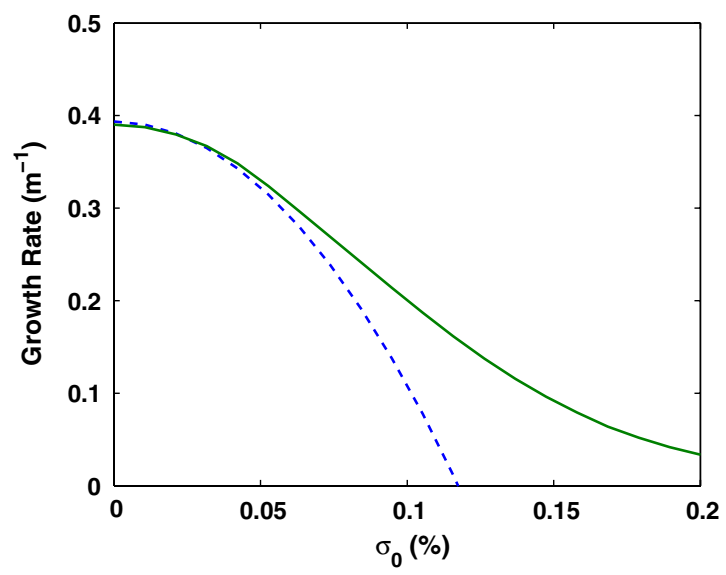

(b)

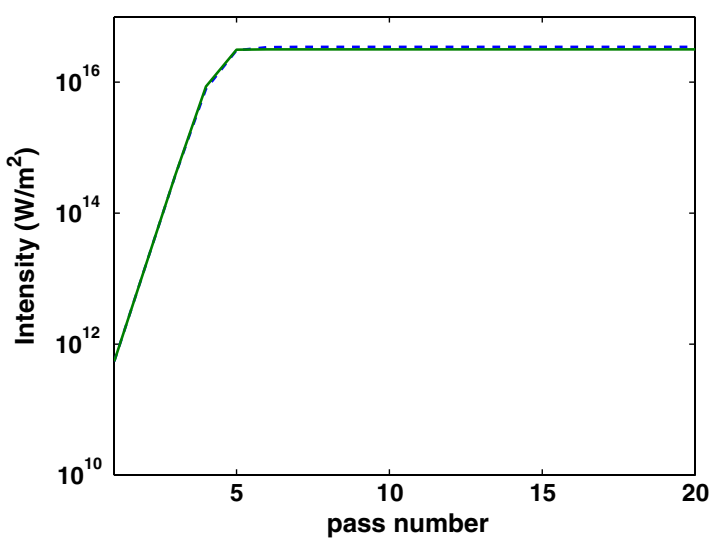

(d)

FIG. 2. A comparison of the logistic model (dashed line) to one-dimensional simulations (solid line) for amplifiers and oscillators with $6.5 \mathrm{~cm}$ undulator period tuned to resonance at $13.4 \mathrm{~nm}$ wavelength: (a) a single, long pass from start-up to saturation; (b) the effect of initial energy spread on growth rate; (c) single-pass simulations for different initial seed power; (d) intensity in a standard oscillator scheme as a function of pass number.

not $\gg 1$. We use $\Sigma_{\text {eff }}$ to convert between power and peak intensity of the radiation.

We treat the effect of diffraction on saturation levels slightly differently from the fit of Ref. [19], which would use $c_{f}\left(\rho_{2}^{2} / \rho\right) P_{\text {beam }}$. That expression is more appropriate for situations dominated by the electron beam energy spread and emittance. Instead, we assume the saturation power scales linearly with the adjusted gain length, $c_{f} \rho_{2} P_{\text {beam }}$, or in terms of intensity $I_{\text {sat }}=c_{f} \rho_{2} P_{\text {beam }} / \Sigma_{\text {eff }}$.

We now consider the effect of an initial relative rms energy spread $\sigma$. When transverse effects are negligible, $\eta_{T} \simeq \sigma^{2} / \rho^{2}$, and when this is smaller than unity we approximate $1 /\left(1+\eta_{T}\right) \simeq 1-\sigma^{2} / \rho^{2}$; this is similar to the leading order term of Ref. [22]. The growth rate [20] is then $\Gamma\left(\sigma_{r}\right)=\left(1-\sigma_{r}^{2} / \rho^{2}\right) \Gamma_{1 \mathrm{D}}$, where $\sigma_{r}$ is the relative energy spread at the end of the radiator section and start of the oscillator. The intensity at saturation [19] is $I_{\text {sat }}\left(\sigma_{r}\right)=$ $\left(1-\sigma_{r}^{2} / \rho^{2}\right)^{2} c_{f} \rho P_{\text {beam }} / \Sigma_{A}$.

We combine these three-dimensional effects in the following way: first, the one-dimensional parameter $\rho$ is replaced by $\rho_{2}$ to account for diffraction. This includes the growth rate for the case of negligible energy spread, the impact of energy spread on the growth rate, and the saturated power. Second, FEL radiation due to electrons in the radiator and oscillator sections is assumed to instantaneously fill a mode with crosssectional area $\Sigma_{\text {eff }}$. The resulting expressions are

$$
\begin{aligned}
\Gamma\left(\sigma_{r}\right) & =\left(1-\sigma_{r}^{2} / \rho_{2}^{2}\right)\left(\rho_{2} / \rho\right) \Gamma_{1 \mathrm{D}}, \\
I_{\text {sat }}\left(\sigma_{r}\right) & =\left(1-\sigma_{r}^{2} / \rho_{2}^{2}\right)^{2} c_{f} \rho_{2} P_{\text {beam }} / \Sigma_{\text {eff }} .
\end{aligned}
$$

We see reasonably good agreement in the growth rate for $\sigma_{r}<\rho_{2} / 2$ as is shown in Fig. 2(b), where $\rho_{2} \approx 1.1 \times 10^{-3}$.

We assume that the oscillator mirrors are curved in such a way as to match into the natural mode size of the FEL. In the radiator, we ignore the fact that it may take a few gain lengths in $z$ for the radiation mode size to approach this value. By contrast, the radiation spot size in the modulator, which has negligible FEL gain, is treated as a free parameter set by the radiation transport optics. There is one constraint on the mode size in the modulator, however; to obtain bunching at the harmonic $n$ with good uniformity across the electron bunch requires a cross-sectional area for the radiation at least $n^{2 / 3}$ times that of the electron beam. 
Otherwise, the reduced intensity near the edges of the electron beam will result in poor bunching there.

The spread in transverse angles in the beam associated with transverse emittance is the one remaining term affecting $\eta_{T}$ but we neglect this effect. The full expression for $\eta_{T}$ calculated by Xie could be used to capture all 3D effects. As the radiator output increases, the average energy of the electron beam entering the oscillator decreases. We do not model the effects of this detuning on the dynamics within the oscillator, and the undulator parameter is assumed to be at the optimal value for the steady state. Temporal variations and slippage between the radiation field and electron beam are also not considered.

\section{Corrections to the linear gain from initial conditions}

In the standard one-dimensional FEL theory, in addition to the growing mode there are two nongrowing eigenmodes as well. To account for this, and the fact that only $\approx 1 / 3$ of the initial field amplitude goes into the growing mode, the initial intensity $I_{0}$ is replaced by $I_{0} / 9$. A slightly improved analysis introduces a gain function $G(z)$ instead of the exponential $\exp (\Gamma z)$ in Eq. (1) to account for the contribution from all three FEL modes [23] given some initial radiation field and an initially uniform (i.e., unbunched) electron distribution:

$$
\begin{aligned}
G(z) & =\frac{1}{9}\left|\exp \left(2 i k_{w} \rho z\right)+2 \cosh \left(\sqrt{3} k_{w} \rho z\right) \exp \left(-i k_{w} \rho z\right)\right|^{2} \\
& =\frac{1}{3}+\frac{1}{9}\left[2 \cosh (\Gamma z)+4 \cos \left(\frac{\sqrt{3} \Gamma z}{2}\right) \cosh \left(\frac{\Gamma z}{2}\right)\right] .
\end{aligned}
$$

Nonideal effects can be approximately included simply by replacing $\rho$ with $\rho_{2}$ and $\Gamma$ with $\Gamma\left(\sigma_{r}\right)$, ignoring small changes in the eigenmodes themselves. This correction is mostly important when the total gain in the undulator is low, or when considering the intensity evolution near the start of the oscillator; for high gain, the growing mode dominates and $G\left(z, \sigma_{r}\right)$ approaches $\exp \left[\Gamma\left(\sigma_{r}\right) z\right] / 9$. Figures 2(a) and 2(c) both show the improved agreement when this correction is employed, especially where the growing mode has not dominated the output intensity in a given pass.

We denote the intensity at the end of the oscillator for a given pass as $I_{k}$ and the energy spread at the beginning of the oscillator as $\sigma_{k}$. At the beginning of the oscillator, the intensity is assumed to be reduced by a factor $R$ to account for mirror losses in the round-trip through the oscillator, outcoupling to the modulator, and any change in spot size between the start and end of the undulator. Putting all of these effects together, our mapping for a pass through an oscillator undulator of length $L$ becomes

$$
I_{k}=R I_{k-1} \frac{G\left(L, \sigma_{k}\right)}{1+\frac{R I_{k-1}}{I_{\mathrm{sat}}\left(\sigma_{k}\right)}\left[G\left(L, \sigma_{k}\right)-1\right]} .
$$

An important parameter for the oscillator is the undulator length measured in ideal gain lengths, $C \equiv \Gamma(\sigma=0) L=2 \sqrt{3} k_{w} \rho L$.

\section{Modeling the HGHG component of the radiator-first configuration}

Now we must determine both the intensity at the end of the radiator, at the harmonic of the oscillator wavelength, and the energy spread $\sigma_{k}$ of the electron beam at the start of the oscillator, based on the intensity $I_{k-1}$ in the previous pass. To do this, we follow the electron beam through the upstream HGHG components using the analytical estimates of Ref. [15]. Where possible, we normalize the oscillator intensity to $I_{\text {ref }} \equiv I_{\text {sat }}(\sigma=0)$, and denote $X_{k}=I_{k} / I_{\text {ref }}$.

Without going into the details of the parameters of the modulator section and radiation transport from the oscillator, the amplitude of the energy modulation generated in the modulator depends linearly on the seed radiation electric field, i.e., on the square root of the intensity. If an intensity $I_{\text {ref }}$ (equivalent to $X=1$ ) coming from the oscillator generates an energy modulation given by $A \sigma_{0}$, where $A$ represents the ratio of the height of this modulation to the original energy spread at the start of the modulator, then for any other value of the intensity the height of the energy modulation will be $A \sigma_{0} \sqrt{X}$. The numerical value of $A$ will depend upon the length of the undulator, the undulator period and strength, the optical transport from the oscillator, and the reference saturation intensity of the oscillator; see Appendix A for a detailed calculation. Typically, we want $A \geq n$, or at least not much smaller than $n$, where $n$ is the desired harmonic upshift. The length of the modulator should also not be much more than two gain lengths long to avoid significant FEL gain and bunching within the modulator, which are ignored in this analysis. The energy spread at the exit of the modulator will then be

$$
\sigma_{m}(X)=\sigma_{0} \sqrt{1+\frac{A^{2}}{2} X}
$$

This modulated beam can be bunched with the use of a simple magnetic chicane. The magnitude of the bunching coefficient for the $n$th harmonic (defined as $b_{n}=\left|\left\langle e^{-i n \theta}\right\rangle\right|$, where $\theta$ is the longitudinal phase of an electron with respect to the oscillator wavelength $\lambda$ ) is given by

$$
b_{n}(X)=e^{-n^{2} B^{2} / 2} J_{n}(n A B \sqrt{X}) .
$$

The parameter $B=2 \pi R_{56} \sigma_{0} / \lambda$ is the dimensionless, scaled dispersion strength of the chicane, where $R_{56}$ is the strength of the dispersion in units of length.

For the radiator section, we assume that FEL amplification stays in the linear regime and never quite reaches saturation, though it will ideally be very close when the system reaches equilibrium. We also assume that there is sufficient total gain that only the growing mode needs to be considered. These simplifying assumptions allows us to use one-dimensional linear theory to find that the radiation intensity at the exit of the radiator will be proportional to the square of its initial bunching. Otherwise, we could use a logistic function to model saturation, and a more general 
gain factor as in Eq. (7) but with different phase factor to match an initial condition of bunching with no initial radiation. We do include the effect of energy spread on FEL gain through the same approximation as was used for the oscillator, to obtain

$$
I_{r}(X)=\frac{1}{9} \rho_{r} I_{\text {beam }, \mathrm{r}}\left|b_{n}(X)\right|^{2} e^{C_{r}\left[1-\sigma_{m}(X)^{2} / \rho_{r}^{2}\right]},
$$

where $\rho_{r}$ is the FEL parameter for the radiator section, $C_{r}=\Gamma_{r}(\sigma=0) L_{r}=2 \sqrt{3} k_{w, r} \rho_{r} L_{r}$ is the undulator length measured in ideal gain lengths, $L_{r}$ is the undulator length in the radiator section, and $k_{w, r}=2 \pi / \lambda_{w, r}$ is the undulator wave number for the radiator section, and $I_{\text {beam,r }}$ is the beam power in the radiator section, which differs from the value in the oscillator only by the ratio of the electron beam cross-sectional area. This result is derived in Appendix B. We note that allowing the radiator to reach saturation might actually improve the stability of the system. The energy spread of the bunch exiting the radiator would then saturate at a value near the FEL parameter in the radiator and would not fluctuate much beyond this point. However, more complex expressions are required to analyze this case.

The induced energy modulation from the radiator is roughly proportional to the square root of the intensity, and the peak amplitude can be written as

$$
2 \rho_{r}|P|=2 \rho_{r} \sqrt{\frac{I_{r}(X)}{\rho_{r} I_{\text {beam }, \mathrm{r}}}}=\frac{2}{3}\left|b_{n}(X)\right| \rho_{r} e^{\left(C_{r} / 2\right)\left[1-\sigma_{m}(X)^{2} / \rho_{r}^{2}\right]} .
$$

This expression is derived in Appendix B.

The energy spread at the exit of the radiator can now be given in terms of the normalized intensity at the end of the oscillator during the previous pass:

$$
\sigma_{r}(X)=\sqrt{\sigma_{m}^{2}(X)+2 \rho_{r} I_{r}(X) / I_{\text {beam }, \mathrm{r}}} .
$$

\section{E. The pass-by-pass map}

In summary, we now have a map for the intensity at the end of the oscillator at pass $k$ in terms of the intensity of the previous pass:

$$
X_{k}=\frac{R X_{k-1} G_{k}}{1+\frac{R X_{k-1}}{\left(1-\sigma_{k}^{2} / \rho^{2}\right)^{2}}\left(G_{k}-1\right)},
$$

where $G_{k}=G\left(L, \sigma_{k}\right)$ is defined by Eq. (7), $\sigma_{k}=$ $\sigma_{r}\left(X_{k-1}\right)$ is defined by Eq. (13), and we have used $X_{\text {sat }}\left(\sigma_{k}\right)=\left(1-\sigma_{k}^{2} / \rho^{2}\right)^{2}$. In all numerical results shown below, we use $\rho_{2}$ (and $\rho_{r 2}$ for the radiator) to correct for the effects of diffraction.

\section{PARAMETERS FOR A SOFT X-RAY EXAMPLE}

Because there are so many constraints that can become important as one moves to different parameter regimes, it is difficult to provide a completely general prescription for designing an HGHG radiator-first scheme. We can, however, mention some guidelines to follow. The electron beam current and brightness, undulator magnet technology, and target radiation wavelength all determine the onedimensional FEL parameter for the radiator section, $\rho_{r}$. The standard emittance constraint applies here, and it is important that the initial energy spread is small enough that the beam will generate the target radiation even after being modulated in the HGHG scheme. The next step is to choose the oscillator wavelength based on magnet technology and mirror availability, which determines the harmonic upshift ratio between the modulator and radiator. The harmonic number must be high enough that $\rho_{r}$ is sufficiently small for the oscillator to work with an initial fractional energy spread of $\rho_{r}$. The harmonic number must also be low enough that the HGHG scheme can produce sufficient bunching to ensure coherence of the output radiation. One can increase the bunching for a given harmonic number by increasing the amplitude of the energy modulation, but this may come at the cost of decreased performance in the radiator due to increased energy spread.

It is clear that one may need to walk a fine line in designing an HGHG radiator-first scheme to meet a particular need, but the map derived in the section above can provide a fast method for numerical parameter scans. We select beam parameters similar to those proposed for a Next Generation Light Source at Lawrence Berkeley National Laboratory [24], with a goal of generating light at around $1 \mathrm{~nm}$. We consider a $2.4 \mathrm{GeV}$ beam with a peak current of $250 \mathrm{~A}$, an initial relative energy spread of $2.9 \times$ $10^{-5}$, and a normalized transverse emittance of $0.3 \mu \mathrm{m}$. We choose a low current because operation at repetition rates above $1 \mathrm{MHz}$ would produce very high average current at high single-bunch charge. With this current and a $4 \mathrm{MHz}$ repetition rate, a $50 \mathrm{pC}$ bunch (roughly $200 \mathrm{fs}$ in duration) would imply an average current of

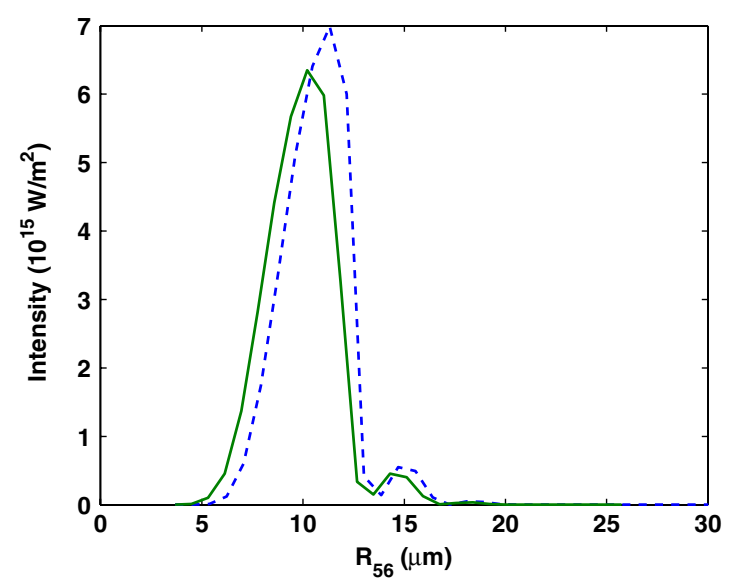

FIG. 3. The map (dashed line) shows qualitative agreement with simulations (solid line) of the saturated intensity from the radiator for a scan of chicane strength. 
$200 \mu \mathrm{A}$. We make use of existing, robust multilayer mirror technology at $13.4 \mathrm{~nm}$ [11] by choosing the resonance wavelength of the oscillator and modulator to equal this value. We can then reach our target wavelength for the output radiation, $1.34 \mathrm{~nm}$, by using the 10th harmonic. The undulator period is $6.5 \mathrm{~cm}$ for the oscillator and modulator, and $2.4 \mathrm{~cm}$ for the radiator. These choices are compatible with a conventional permanent magnet design with a magnetic gap of over $8 \mathrm{~mm}$. For simplicity, we have chosen the average beta functions to be compatible with the use of natural undulator focusing with curved pole faces for equal focusing in $x$ and $y$. For the oscillator in particular, diffraction will limit the benefits to decreasing the spot size of the electron beam. For the radiator, it could be useful to use stronger focusing to increase the power output so long as it does not lead to an energy spread at the end of the radiator so high as to significantly weaken the oscillator perform-

TABLE I. Some key electron beam parameters.

\begin{tabular}{lc}
\hline \hline Bunch charge & $50 \mathrm{pC}$ \\
Nominal energy & $2.4 \mathrm{GeV}$ \\
Slice energy spread & $70 \mathrm{keV}$ \\
Slice transverse emittance & $0.3 \mu \mathrm{m}$ \\
Current & $250 \mathrm{~A}$ \\
Repetition rate & $4 \mathrm{MHz}$ \\
\hline \hline
\end{tabular}

ance. The electron beam size in the modulator is assumed to match that of the radiator.

The ratio of the one-dimensional FEL parameter of the oscillator to that of the radiator is 2.6. Thus, in the onedimensional approximation we can guarantee that the energy spread entering the oscillator will be $<0.4 \rho$ when the HGHG radiation generation is saturated. When diffraction is taken into account, this ratio drops to 2.1. In either case, this should ensure that the beam quality is sufficient for the oscillator to replenish the roughly $50 \%$ radiation fraction lost from the mirrors and outcoupling to the modulator.

We design the modulator so that it produces a modulation of less than $7 \sigma_{0}$ to prevent energy spread from limiting the output of the radiator. With these parameters, we can expect a maximum bunching of almost $10 \%$ at the 10th harmonic, which is sufficient to generate good longitudinal coherence. The strength of the chicane in the HGHG scheme can be estimated [15], but the exact value was determined by scanning through values of $B$ in the map to maximize the radiator output. Figure 3 shows qualitative agreement of this scan to the one-dimensional simulation. There is a small shift in $R_{56}$ due to the model missing some dispersion generated by the undulators themselves. The electron beam and beam line parameters are given in Tables I and II. The translation of these parameters into the constants used by the map is shown in Table III.

TABLE II. Some key parameters for the beam line elements.

\begin{tabular}{lccccccc}
\hline \hline Element & $R_{56}$ & $\lambda$ & $\lambda_{w}$ & $a_{w}$ & \# Periods & Reflectivity $R$ & Transmission $T$ \\
\hline $\begin{array}{l}\text { Modulator } \\
\text { First chicane }\end{array}$ & $11.5 \mu \mathrm{m}$ & $13.4 \mathrm{~nm}$ & $6.5 \mathrm{~cm}$ & 2.8 & 42 & & \\
$\begin{array}{l}\text { Radiator } \\
\text { Second chicane }\end{array}$ & $1000 \mu \mathrm{m}$ & $1.34 \mathrm{~nm}$ & $2.4 \mathrm{~cm}$ & 1.2 & 600 & & 0.02 \\
\begin{tabular}{l} 
Oscillator \\
\hline \hline
\end{tabular} & & $13.4 \mathrm{~nm}$ & $6.5 \mathrm{~cm}$ & 2.8 & 240 & 0.49 & 0.02 \\
\hline
\end{tabular}

TABLE III. A summary of the parameters used in the map. These values were calculated from the machine parameters as explained in this paper.

\begin{tabular}{lcl}
\hline \hline Parameter & Value & \multicolumn{1}{c}{ Physical interpretation } \\
\hline$I_{\text {ref }}$ & $3.5 \times 10^{16} \mathrm{~W} / \mathrm{m}^{2}$ & Reference saturation intensity for oscillator undulator \\
$I_{\text {beam,r }}$ & $1.1 \times 10^{20} \mathrm{~W} / \mathrm{m}^{2}$ & Electron beam power density in radiator section \\
$\sigma_{0}$ & $2.9 \times 10^{-5}$ & Initial fractional energy spread of electron beam \\
$\rho$ & $1.7 \times 10^{-3}$ & One-dimensional FEL parameter in oscillator \\
$\rho_{2}$ & $1.1 \times 10^{-3}$ & FEL parameter in oscillator corrected for diffraction \\
$\rho_{r}$ & $6.5 \times 10^{-4}$ & One-dimensional FEL parameter in radiator \\
$\rho_{r 2}$ & $5.4 \times 10^{-4}$ & FEL parameter in radiator corrected for diffraction \\
$n$ & 10 & Harmonic upshift between oscillator and radiator \\
$A$ & 8.19 & Reference energy modulation amplitude after modulator, \\
& & in units of $\sigma_{0}$, when $I_{\text {ref exits oscillator during previous pass }}$ \\
$B$ & 0.156 & Scaled dispersion strength of chicane after modulator \\
$C$ & 6.14 & Length of oscillator undulator in gain lengths (with diffraction) \\
$C_{r}$ & 6.44 & Length of radiator undulator in gain lengths (with diffraction) \\
$R$ & 0.49 & Power reflectivity for round-trip in oscillator \\
$T$ & 0.02 & Net outcoupling of power from end of oscillator to modulator \\
\hline \hline
\end{tabular}




\section{COMPARISON OF THE MAP TO ONE-DIMENSIONAL SIMULATIONS}

The system was simulated with an implementation of the simplified FEL equations (see, for example, [25]) in MATLAB. The one-dimensional equations to be solved are

$$
\begin{gathered}
\frac{d \theta_{j}}{d \bar{z}}=\bar{\eta}_{j}, \\
\frac{d \bar{\eta}_{j}}{d \bar{z}}=\tilde{a} e^{i \theta_{j}}+\tilde{a}^{*} e^{-i \theta_{j},} \\
\frac{d \tilde{a}}{d \bar{z}}=-\left\langle e^{-i \theta}\right\rangle,
\end{gathered}
$$

where we use scaled variables

$$
\begin{gathered}
\theta=\left(k+k_{w}\right) z-c k t, \\
\bar{\eta}=\frac{\gamma-\gamma_{0}}{\rho \gamma_{0}}=\frac{\eta}{\rho}, \\
\tilde{a}=\frac{\alpha}{\rho^{2}} \frac{e \tilde{E}}{\sqrt{2} k m_{e} c^{2}}, \\
\bar{z}=2 k_{w} \rho z,
\end{gathered}
$$

and where $\alpha=k a_{w}[J J] / 2 \gamma_{0}^{2} k_{w}$ and $\tilde{E}$ is the complex electric field amplitude of the radiation on axis. Using the resonance condition, the quantity $\alpha$ appears in the expression for the FEL parameter, yielding the formula

$$
\frac{1}{\alpha^{2}}=\frac{1}{\rho^{3}} \frac{1}{4 \pi} \frac{I_{e}}{I_{A}} \frac{\gamma_{0} \lambda^{2}}{\Sigma_{A}}
$$

To take into account the effect of diffraction, the model equation for the radiation field, Eq. (17), must be modified to include the difference between the expected size of the radiation mode and that of the electron beam, yielding

$$
\frac{d \tilde{a}}{d \bar{z}}=-\left\langle e^{-i \theta}\right\rangle \frac{\Sigma_{A}}{\Sigma_{\mathrm{eff}}} .
$$

The equations of motion for $\theta_{j}$ and $\eta_{j}$ are unchanged.

Our simulations use only a single electron beam and radiation slice, corresponding to a time-steady, monochromatic case. Thus, polychromatic nonzero bandwidth and slippage effects within the bunch, which are not captured by our map, do not enter into our simulations. We include analytical dispersive maps to propagate the electron beam through the chicane and to model the

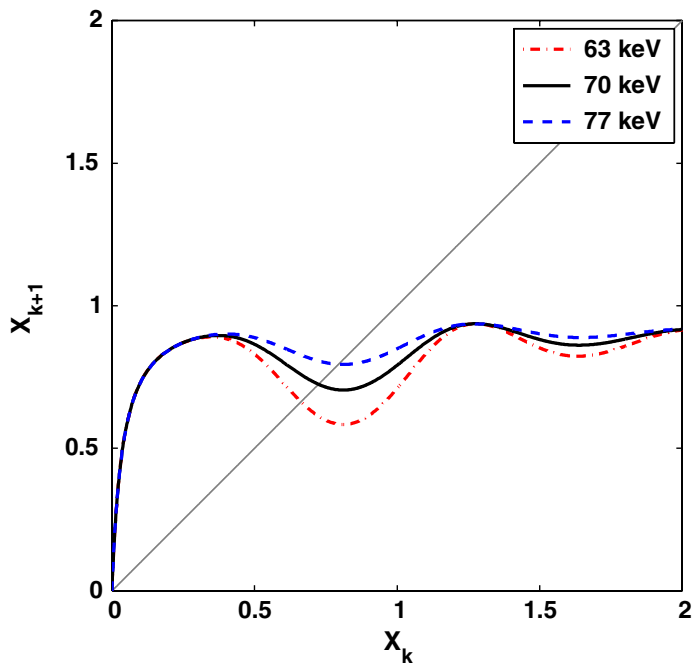

FIG. 4. Normalized intensity at the end of the oscillator as a function of that of the previous pass. Three values of the initial energy spread are considered, with all other parameters held fixed. The diagonal line indicates possible equilibrium points. For the nominal $70 \mathrm{keV}$ case, the slope of the map at the equilibrium point is -0.45 . All three cases are stable.

beam propagation subsequent to the radiator. To match our assumptions, we have chosen the dispersion after the radiator to have a value of $\sim 1 \mathrm{~mm}$, which is strong enough to remove virtually all bunching remaining from the modulation section. For both the simulations and the study using the map, we use an initial power level in the oscillator of $c \rho^{2} / \lambda$ times the electron energy, as explained in Ref. [25].

With the parameters for our soft x-ray example, we plot the intensity at the exit of the oscillator as a function of the intensity at the end of the previous pass in Fig. 4, as the black solid curve. The intersection of the map with the line $X_{k}=X_{k-1}$ is an equilibrium point. Furthermore, if the absolute value of the slope at the equilibrium is less than one, it is a stable equilibrium point. With the above parameters, the equilibrium point occurs at $X=0.723$ which corresponds to an intensity of $2.5 \times 10^{16} \mathrm{~W} / \mathrm{m}^{2}$. The slope of the map at this equilibrium point is -0.45 , indicating that there should be damped oscillations about this point. The chicane strength after the modulation section is adjusted slightly below the dispersion for maximum bunching at equilibrium; this provides negative feedback against fluctuations in oscillator power, as might occur when better performance of the radiator section leads to more energy spread and reduced oscillator gain. The steady-state power produced by the radiator is a factor of roughly 0.20 times that of the ideal saturated power level. Also shown are the equivalent results for when the energy spread is decreased and increased by $10 \%$, all other parameters being kept fixed. The case of reduced initial energy spread, with 


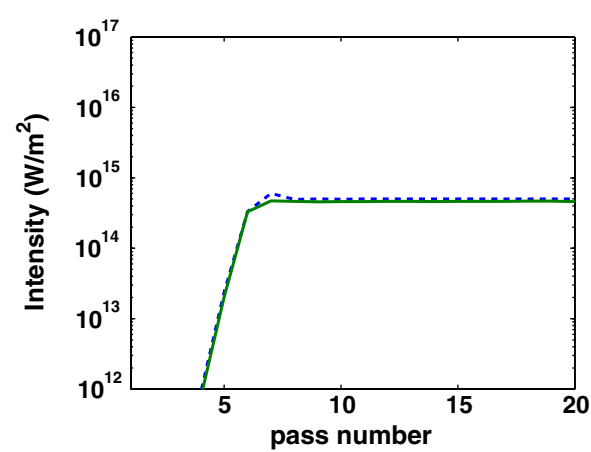

(a)

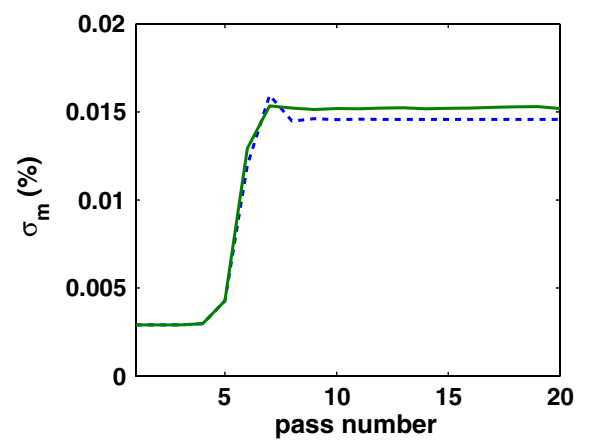

(c)

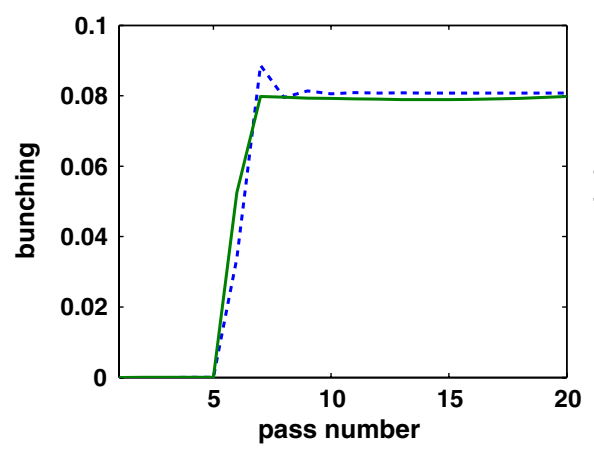

(e)

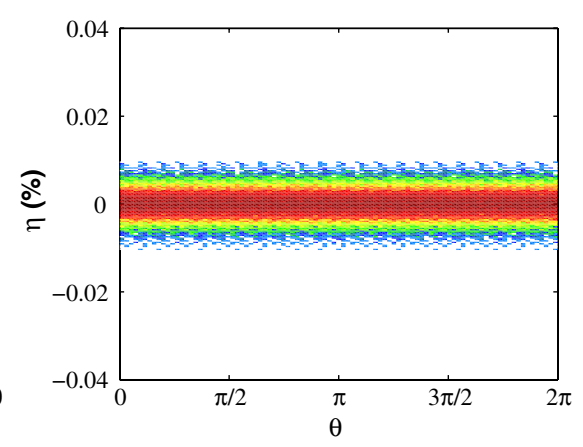

(b)

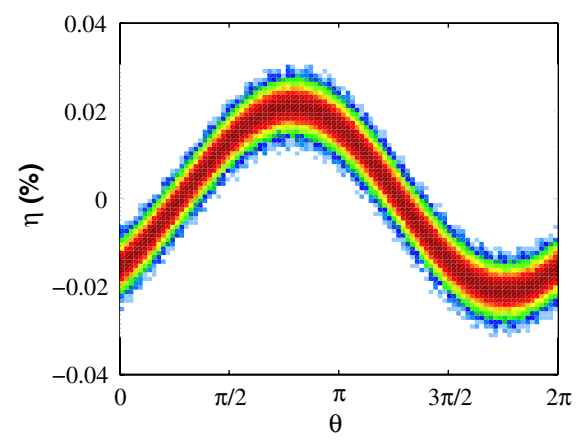

(d)

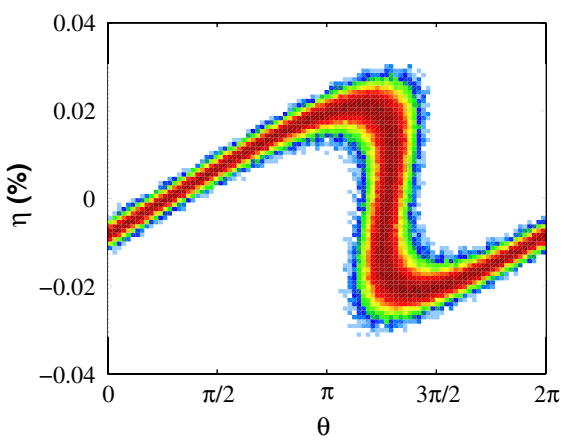

(f)

FIG. 5. A comparison of the map (dashed line) to a one-dimensional simulation (solid line), and equilibrium longitudinal phase spaces for key points along the prebunching section of the HGHG radiator-first scheme with red areas indicating higher phase space density: (a) intensity of the $13.4 \mathrm{~nm}$ seed radiation reaching the modulation section; (b) initial phase space of the electron beam; (c) energy spread at the exit of the modulator; (d) phase space at the end of the modulator; (e) bunching at the 10th harmonic after the chicane; and (f) phase space after chicane.

its corresponding improved bunching entering the radiator, is close to being unstable with a slope of -0.98 . Attempting to further increase the ratio of steady-state power out of the radiator to the ideal saturated power level tends to drive the equilibrium point unstable.

Figures 5 and 6 show good agreement between the map and simulation from start-up through saturation at key locations along the scheme. The corresponding longitudinal phase space of the electron beam at saturation (pass 20) is also shown for each location, truncated to only show regions with $>1 \%$ of the peak density. The overall performance at equilibrium for these parameters is given in Table IV for both wavelengths. These values, in terms of peak power and intensity, are also compared to the nominal saturation values for the given electron beam parameters. The ratio of the equilibrium power to saturated power in the radiator is 0.14 in the simulations, compared to 0.20 for the nonlinear map. However, part of this difference is explained by the offset in optimal $R_{56}$ seen in Fig. 3, and changing $R_{56}$ from 11.5 to $10.5 \mu \mathrm{m}$ yields a ratio of 0.18 while the range of energy spreads for which the system is stable is similar to that for the map. This is a more meaningful measure of the discrepancy between the map and numerical simulation. 


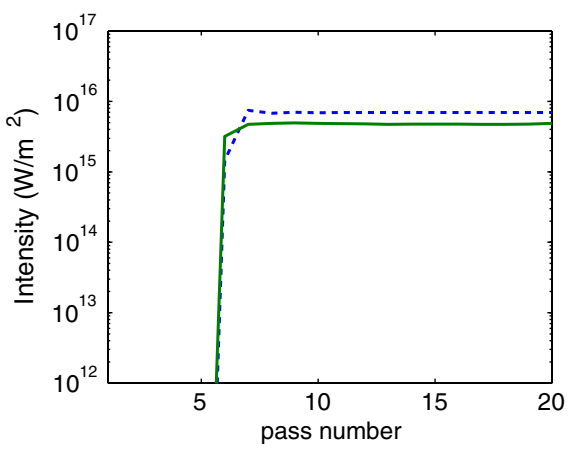

(a)

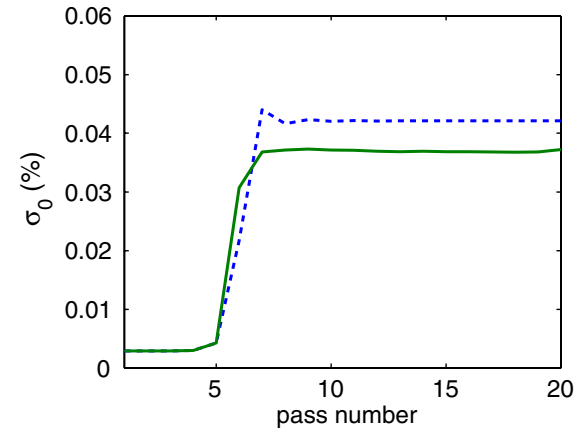

(c)

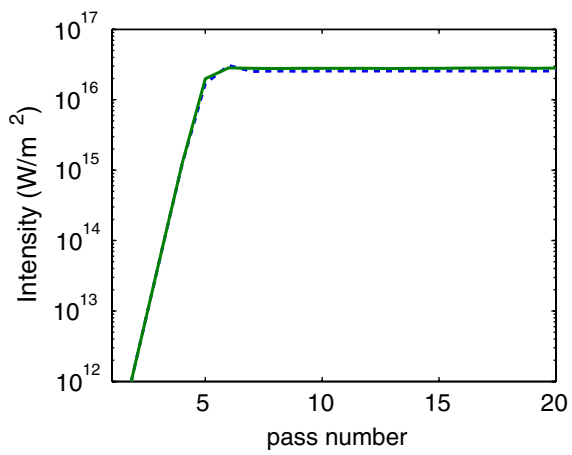

(e)

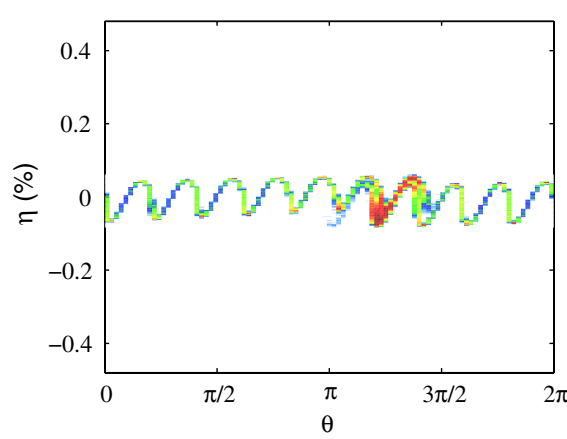

(b)

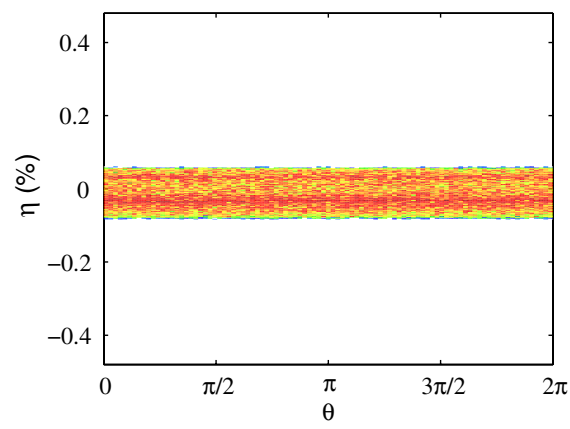

(d)

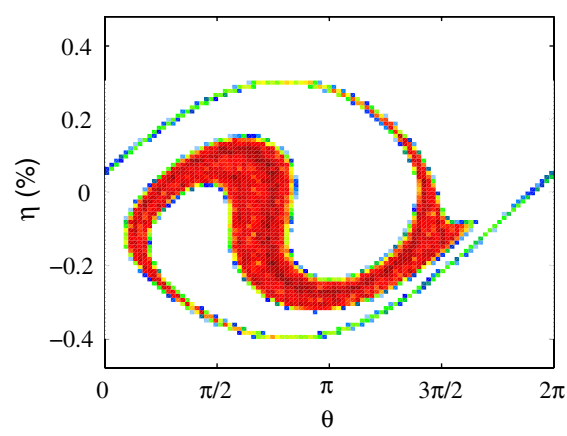

(f)

FIG. 6. A comparison of the map (dashed line) to a one-dimensional simulation (solid line), and equilibrium longitudinal phase spaces for key points along the target radiation generation and oscillator sections of the radiator-first scheme with red areas indicating higher phase space density. Note that the energy scale for the phase space diagrams here is $12 \times$ larger than for those of Fig. 5 : (a) intensity of radiation at $1.34 \mathrm{~nm}$ exiting radiator; (b) phase space at the end of the radiator; (c) energy spread entering the oscillator; (d) phase space entering the oscillator; (e) intensity of $13.4 \mathrm{~nm}$ radiation at the end of the oscillator; and (f) phase space at the end of the oscillator.

TABLE IV. Performance of the radiator-first beam line in equilibrium, and compared to nominal saturation values.

\begin{tabular}{lccccc}
\hline \hline & & \multicolumn{2}{c}{ Power } & \multicolumn{2}{c}{ Intensity } \\
Element & Radiation spot size & Equilibrium & Saturation & Equilibrium & Saturation \\
\hline Oscillator & $71 \mu \mathrm{m}$ & $888 \mathrm{MW}$ & $1130 \mathrm{MW}$ & $2.8 \times 10^{16} \mathrm{~W} / \mathrm{m}^{2}$ & $3.5 \times 10^{16} \mathrm{~W} / \mathrm{m}^{2}$ \\
Modulator & $78 \mu \mathrm{m}$ & $17 \mathrm{MW}$ & $22 \mathrm{MW}$ & $4.6 \times 10^{14} \mathrm{~W} / \mathrm{m}^{2}$ & $5.8 \times 10^{14} \mathrm{~W} / \mathrm{m}^{2}$ \\
Radiator & $47 \mu \mathrm{m}$ & $66 \mathrm{MW}$ & $474 \mathrm{MW}$ & $4.9 \times 10^{15} \mathrm{~W} / \mathrm{m}^{2}$ & $3.5 \times 10^{16} \mathrm{~W} / \mathrm{m}^{2}$ \\
\hline \hline
\end{tabular}




\section{CONCLUSIONS}

We have numerically and analytically studied the radiator-first FEL configuration. The coupled HGHG radiator and oscillator system should allow for the production of longitudinally coherent pulses at repetition rates above $1 \mathrm{MHz}$. By targeting different harmonics of the oscillator for the output radiation, this scheme can even provide some level of tunability, although the useful reflecting bandwidth of multilayer mirrors in this wavelength range is presently too narrow to bridge the gap between discrete harmonics. The parameters of these simulations are targeted toward a soft $\mathrm{x}$-ray FEL, but this scheme could be implemented in any regime where a high repetition rate is necessary and appropriate optics are available. At present, multilayer mirrors with high reflectivity are restricted to less than $100 \mathrm{eV}$ photon energy. For photon energies above $\sim 2 \mathrm{keV}$, Bragg scattering provides a different mechanism for the oscillator optics.

We have found that a semianalytic nonlinear map, based on well-known one-dimensional, time-independent FEL approximations, yields an understanding of the dynamics from start-up to saturation and stable operation. The theory does not consider key questions such as finite pulse effects (e.g., slippage), transverse variations of optical fields, $x$ rays, or the particle beam, and pulse-to-pulse jitter. Consideration of these effects together with twodimensional time-dependent simulations are necessary for a detailed understanding of the usefulness of this scheme. Further analytic progress can be anticipated in specific regimes; for example, the work of Piovella et al., [16] may provide a path to a more robust analytic theory for the case where the electron bunch is short compared to the slippage within the oscillator. For proper inclusion of slippage and 3D effects, results from these methods should be corroborated by the use of a code such as GINGER [26] which can directly model oscillators. The approach presented here can be extended to the EEHG radiator-first scheme discussed in [9], or to other harmonic generation schemes that require seed radiation from an external laser.

\section{ACKNOWLEDGMENTS}

This work was supported by the Director, Office of Science, Office of Basic Energy Sciences, of the U.S. Department of Energy under Contracts No. DE-AC0205CH11231 and No. DE-FG02-06CH11357.

\section{APPENDIX A: DERIVATION OF FORMULA FOR MODULATION PARAMETER $\boldsymbol{A}$}

The subject of this Appendix is the calculation of the amplitude of the energy modulation at the end of the modulator. The starting point for the calculation is the set of equations given in Eqs. (15)-(17). The authors of the reference from which those equations came introduce collective variables as follows:

$$
\begin{gathered}
b=\left\langle e^{-i \theta}\right\rangle, \\
P=\left\langle\bar{\eta} e^{-i \theta}\right\rangle .
\end{gathered}
$$

The linearized differential equations for the collective variables are

$$
\begin{gathered}
\frac{d \tilde{a}}{d \bar{z}}=-b, \\
\frac{d b}{d \bar{z}}=-i P, \\
\frac{d P}{d \bar{z}}=\tilde{a} .
\end{gathered}
$$

For the case of the modulator, we have nonzero $\tilde{a}(0)$, while $b(0)$ and $P(0)$ are both presumed zero. Since the length of the modulator is less than or close to a gain length, we can approximate the solution by ignoring FEL gain in the field, taking $\tilde{a}$ to be constant to obtain

$$
P(\bar{z})=\tilde{a}(0) \bar{z} .
$$

An elementary calculation can be used to show that for a modulated beam, $\left|\left\langle\eta e^{-i \theta}\right\rangle\right|$ is equal to half the modulation amplitude on the $\eta$ axis, independent of the value of the initial energy spread. The notation used in this paper is that $A$ values are used for modulation amplitude on the $\eta$ axis divided by $\sigma_{0}$. Thus, we have

$$
\left|\left\langle\eta e^{-i \theta}\right\rangle\right|=\frac{1}{2} A \sigma_{0} .
$$

Because $P$ is equal to $\left\langle\eta e^{-i \theta}\right\rangle$ divided by $\rho$, Eq. (A6) implies

$$
\rho|\tilde{a}(0)| \bar{z}=\frac{1}{2} A \sigma_{0},
$$

which can be written as

$$
A=\frac{4 k_{w} \rho^{2} L_{m}}{\sigma_{0}}|\tilde{a}(0)|,
$$

where $L_{m}$ is the total length of undulator in the modulator section.

The time-averaged (over an optical cycle) intensity, which is the density of power flux in the electromagnetic field, is [25]

$$
I=2 \epsilon_{0} c|\tilde{E}|^{2}=|\tilde{a}(0)|^{2} 2 \epsilon_{0} c \frac{2 \rho^{4} k^{2} m_{e}^{2} c^{4}}{\alpha^{2} e^{2}} .
$$

Using Eq. (22) and the definition of $I_{A}$, this can be simplified to

$$
I=|\tilde{a}(0)|^{2} \rho \frac{\gamma_{0} m_{e} c^{2} I_{e}}{e \Sigma_{A}}=|\tilde{a}(0)|^{2} \rho I_{\text {beam }},
$$

which can be written as 


$$
|\tilde{a}(0)|=\sqrt{\frac{I}{\rho I_{\text {beam }}}} .
$$

Thus, from Eq. (A9) we have

$$
A=\frac{4 k_{w} L_{m}}{\sigma_{0}} \sqrt{\frac{\rho^{3} I(0)}{I_{\text {beam }}}},
$$

where $I(0)$ is the intensity at the beginning of the modulator (which actually does not change as a function of $z$ in this approximation). Because the quantity $A$ is defined to be the scaled modulation for the case of the reference intensity $I_{\text {ref }}$ at the end of the oscillator, we substitute in the value $I(0)=T I_{\text {ref }}$.

Note that we have assumed identical undulator and electron beam parameters in the oscillator and modulator sections. While the undulator parameters should be similar, because the radiation wavelength is the same, the radiation spot size could be different. However, both $\rho^{3}$ and $I_{\text {beam }}$ scale as $1 / \Sigma_{A}$, so for low gain the results are insensitive to the bulk properties of the electron beam.

\section{APPENDIX B: DERIVATION OF FORMULAS FOR THE RADIATOR SECTION}

The subject of this Appendix is the calculation of the radiation intensity and beam energy spread at the end of the radiator section. The amplitude of the energy modulation and intensity of the radiation field must be known in order to evaluate the performance of the entire scheme. The exact solution of the one-dimensional system of differential equations for the collective variables is well known [25], especially in the exponential-growth regime where the growing mode dominates. For the case of the radiator, the only nonzero quantity at the start of the undulators is the bunching parameter $b(0)$; both $\tilde{a}(0)$ and $P(0)$ are presumed zero. The solution for the growing mode is

$$
\begin{gathered}
\tilde{a}(\bar{z})=-i \frac{b(0)}{3} \mu_{+}^{2} e^{-i \mu_{+} \bar{z}}, \\
b(\bar{z})=\frac{b(0)}{3} e^{-i \mu_{+} \bar{z}} \\
P(\bar{z})=\frac{b(0)}{3} \mu_{+} e^{-i \mu_{+} \bar{z}},
\end{gathered}
$$

where $\mu_{+}=-1 / 2+i \sqrt{3} / 2$ is one of the cube roots of 1 . These expressions clearly satisfy Eqs. (A3)-(A5). The other two modes are phased differently from the case in the oscillator, due to the different initial conditions. To roughly account for the effect of energy spread, we replace the one-dimensional gain length with the corrected gain length, but ignore any changes in the nature of the mode solutions, which may affect the relative weight of the growing mode.
At the end of the radiator, which is presumed long compared to the gain length, the magnitude of the scaled radiation field is

$$
\left|\tilde{a}\left(L_{r}\right)\right|=\left|\frac{b(0)}{3}\right| e^{C_{r} / 2},
$$

where $C_{r}=2 \sqrt{3} k_{w} \rho_{r} L_{r}$ and the subscript $r$ refers to properties in the radiator section. The effect of nonzero energy spread is roughly to make one replace $C_{r}$ with $C_{r}\left(1-\sigma_{m}^{2} / \rho_{r}^{2}\right)$. The scaling law of Eq. (A12) still applies to the radiator, but with different scaled variables:

$$
\left|\tilde{a}\left(L_{r}\right)\right|=\sqrt{\frac{I_{r}}{\rho_{r} I_{\text {beam }, \mathrm{r}}}} .
$$

This yields

$$
I_{r}=\left|\tilde{a}\left(L_{r}\right)\right|^{2} \rho_{r} I_{\text {beam }, \mathrm{r}}=\left|\frac{b(0)}{3}\right|^{2} e^{C_{r}} \rho_{r} I_{\text {beam }, \mathrm{r}} .
$$

Allowing for an increased gain length,

$$
I_{r}=\left|\frac{b(0)}{3}\right|^{2} e^{C_{r}\left(1-\sigma_{m}^{2} / \rho_{r}^{2}\right)} \rho_{r} I_{\text {beam }, \mathrm{r}} .
$$

The height of the energy modulation is still given by

$2 \rho_{r}|P|=2 \rho_{r}|b(0) / 3| e^{\left(C_{r} / 2\right)\left(1-\sigma_{m}^{2} / \rho_{r}^{2}\right)}=2 \rho_{r} \sqrt{I_{r} / \rho_{r} I_{\text {beam }, \mathrm{r}}}$,

substituting in the solution from Eq. (B7). In the linear regime, the incoherent energy spread at the end of the radiator is given by

$$
\sigma_{r}^{2}=\sigma_{m}^{2}+2 \rho_{r}^{2}|P|^{2}=\sigma_{m}^{2}+2 \rho_{r} I_{r} / I_{\text {beam }, \mathrm{r}} .
$$

When diffraction is taken into account, in addition to correcting the value of $\rho_{r}$, the cross-sectional area of the electron beam should be replaced by the area of the radiation mode, so that $I_{\text {beam,r }}$ should be replaced by $I_{\text {beam }, \mathrm{r}} \Sigma_{A, r} / \Sigma_{\text {eff,r }}$.

[1] G. R. Neil et al., Nucl. Instrum. Methods Phys. Res., Sect. A 557, 9 (2006).

[2] "Realizing the Potential of Seeded FELs in the Soft X-Ray Regime" [https://sites.google.com/a/lbl.gov/ realizing-the-potential-of-seeded-fels-in-the-soft-X-rayregime-workshop/].

[3] G. Geloni, V. Kocharyan, and E. Saldin, arXiv:1003.2548v1.

[4] L. H. Yu, Phys. Rev. A 44, 5178 (1991).

[5] G. Stupakov, Phys. Rev. Lett. 102, 074801 (2009).

[6] J. Amann, W. Berg, V. Blank, F.-J. Decker et al., Nat. Photonics 6, 693 (2012).

[7] D. J. Bamford and D. A. G. Deacon, Nucl. Instrum. Methods Phys. Res., Sect. A 304, 667 (1991).

[8] G. Dattoli, L. Giannessi, P. L. Ottaviani, Nucl. Instrum. Methods Phys. Res., Sect. A 507, 26 (2003). 
[9] J. Wurtele et al., in Proceedings of the 2010 FEL Conference (Malmö, Sweden, 2010), TUOCI2, pp. 315-321.

[10] G. Penn and M. Reinsch, J. Mod. Opt. 58, 1404 (2011).

[11] S. M. Al-Marzoug and R. J. W. Hodgson, Appl. Opt. 47, 2155 (2008).

[12] R. Barbini et al, in Proceedings of the Workshop on Prospects for a $1 \AA$ Free-Electron Laser (Sag Harbor, NY, 1990), BNL Report No. 52273, 1991, pp. 57-62; F. Ciocci, G. Dattoli, A. De Angelis, B. Faatz, F. Garosi, L. Giannessi, P. L. Ottaviani, and A. Torre, IEEE J. Quantum Electron. 31, 1242 (1995).

[13] G. Dattoli, B. Faatz, L. Giannessi, and P. L. Ottaviani, IEEE J. Quantum Electron. 31, 1584 (1995).

[14] G. Dattoli, L. Giannessi, P. L. Ottaviani, and M. Carpanese, Nucl. Instrum. Methods Phys. Res., Sect. A 393, 133 (1997).

[15] L. H. Yu and J. Wu, Nucl. Instrum. Methods Phys. Res., Sect. A 483, 493 (2002).

[16] N. Piovella, P. Chaix, G. Shvets, and D. A. Jaroszynski, Phys. Rev. E 52, 5470 (1995).

[17] W. Colson, Laser Handbook (North-Holland, Amsterdam, 1990), Vol. 6, Chap. 5.
[18] G. Dattoli, J. Appl. Phys. 84, 2393 (1998).

[19] K.-J. Kim and M. Xie, Nucl. Instrum. Methods Phys. Res., Sect. A 331, 359 (1993).

[20] M. Xie, Nucl. Instrum. Methods Phys. Res., Sect. A 445, 59 (2000).

[21] Analytic calculations for diffraction-dominated beams reveal that there is in fact a weak, logarithmic singularity in the growth rate as the electron beam radius becomes small; see G. T. Moore, Nucl. Instrum. Methods Phys. Res., Sect. A 239, 19 (1985).

[22] G. Dattoli, L. Giannessi, P. L. Ottaviani, and C. Ronsivalle, J. Appl. Phys. 95, 3206 (2004).

[23] G. Dattoli, P. L. Ottaviani, and S. Pagnutti, J. Appl. Phys. 97, 113102 (2005).

[24] J.N. Corlett et al., in Proceedings of the 2010 FEL Conference (Malmö, Sweden, 2010), MOPA06, pp. 38-40.

[25] Z. Huang and K.-J. Kim, Phys. Rev. ST Accel. Beams 10, 034801 (2007).

[26] W. M. Fawley, LBNL Report No. LBNL-49625-Rev.1, 2004 [http://www-ssrl.slac.stanford.edu/lcls/technotes/ LCLS-TN-04-3.pdf]. 